\title{
Puerarin inhibits FUNDC1-mediated mitochondrial autophagy and CSE-induced apoptosis of human bronchial epithelial cells by activating the PI3K/AKT/mTOR signaling pathway
}

\author{
Li Wang ${ }^{1, *}$, Weizhou Jiang ${ }^{2,}{ }^{*}$, Jing Wang ${ }^{3}$, Yuanyuan Xie ${ }^{4}$, Weisi Wang ${ }^{5}$ \\ ${ }^{1}$ Department of Respiratory Medicine, Yan'an University Affiliated Hospital, Yan'an 716000, China \\ ${ }^{2}$ Department of Pulmonology, Weifang Traditional Chinese Hospital, Weifang 261041, China \\ ${ }^{3}$ Endoscopy Room, Tai'an Maternal and Child Health Hospital, Tai'an 271000, China \\ ${ }^{4}$ Department of Geriatrics, Yan'an University Affiliated Hospital, Yan'an 716000, China \\ ${ }^{5}$ Department of Respiratory Medicine, The Second Affiliated Hospital of Zhejiang Chinese Medical University, \\ Hangzhou 310005, China \\ *Equal contribution
}

Correspondence to: Weisi Wang; email: wangweisi@zcmu.edu.cn

Keywords: chronic obstructive pulmonary disease, puerarin, mitochondrial autophagy, FUNDC1, PI3K/AKT/mTOR signaling pathway

Received: January 7, $2021 \quad$ Accepted: March 27, $2021 \quad$ Published: February 8, 2022

Copyright: (C) 2021 Wang et al. This is an open access article distributed under the terms of the Creative Commons Attribution License (CC BY 3.0), which permits unrestricted use, distribution, and reproduction in any medium, provided the original author and source are credited.

\section{ABSTRACT}

Increasing evidence suggests that the pathogenesis of chronic obstructive pulmonary disease (COPD) is associated with FUN14 domain protein 1 (FUNDC1)-mediated mitophagy. Recently, studies have reported that puerarin has protective effects against excessive oxidative damage in cells. Therefore, we hypothesized that puerarin may be involved in COPD progression via regulating FUNDC1 mediated mitophagy. We found that the viability of cigarette smoke extract (CSE)-stimulated human bronchial epithelial cells (HBECs) was enhanced and apoptosis was reduced after treatment with different concentrations of puerarin. Puerarin reversed mitochondrial membrane potential (MMP) levels and ATP content, and decreased reactive oxygen species (ROS) content in CSE stimulated HBECs. Moreover, puerarin significantly inhibited apoptosis related proteins, as well as the expression of mitophagy related proteins. After inhibition of FUNDC1 phosphorylation by protein phosphatase inhibitor (PH0321), puerarin restored MMP level, decreased ROS content, promoted ATP synthesis, and downregulated autophagy related protein expression in HBECs. In addition, mitochondrial division inhibitor (Mdivi) inhibited the expression of autophagy related proteins and reduced apoptosis after blocking cell autophagy, which was the same as the inhibition of puerarin. Finally, puerarin activated the PI3K/Akt/mTOR signaling pathway to participate in COPD progression by up regulating the phosphorylation levels of PI3K, Akt and mTOR.

\section{INTRODUCTION}

Chronic obstructive pulmonary disease (COPD) is a common disease characterized by persistent airflow limitation, and its clinical manifestations are mainly chronic cough, shortness of breath, chest tightness or wheezing $[1,2]$. Persistent chronic inflammation induces the recurrence of tracheal wall injury and repair process, leading to airway remodeling, which is the main cause of irreversible progression of COPD $[3,4]$. The morbidity and mortality of COPD are increasing year by year, which seriously affects the 
working ability and quality of life of patients and causes a heavy socio-economic burden.

Smoking is the most important risk factor for COPD [5]. Cigarette smoke constantly stimulates the alveoli, leading to the elevation of endogenous reactive oxygen species (ROS) in inflammatory cells and epithelial cells, and mitochondrial damage, which in turn induces the imbalance between oxidative and antioxidant processes in cells [6]. It has been shown that cigarette smoke extract (CSE)-stimulated human bronchial epithelial cells (HBECs) exhibit stronger mitophagy $[7,8]$. In COPD progression, mammalian mitotic receptor FUN14 domain protein 1 (FUNDC1) is closely related to autophagy and apoptosis in hypoxic cells, which can participate in mitosis through enhanced mitophagy [9]. Wen et al. found that silencing the FUNDC1 gene in CSE-stimulated HBECs, the expression of DRP1 was also inhibited, thus inhibiting mitophagy and apoptosis and slowing down the COPD process [10].

At present, most of the acute treatment options for COPD are antibiotics, bronchodilators, glucocorticoids and expectorants, but these treatment measures could not effectively delay or prevent the recurrence and progressive progress of the disease. Studies have shown that flavonoids have anti-inflammatory and antioxidant properties, which can effectively improve lung function and reduce inflammatory cell infiltration in chronic airway inflammation [11-13]. Gao et al. showed that Icariside II could protect PC12 cells from $\mathrm{H}_{2} \mathrm{O}_{2}$-induced death by inhibiting mitochondrialmediated autophagy [14].

Puerarin is an isoflavone compound isolated from the dried root of Pueraria lobata, a traditional Chinese medicine. Its medicinal value is benefited from its wide range of pharmacological properties, such as vasodilation, cardio-protection, antioxidant, antiapoptotic and reducing insulin resistance [15]. Puerarin plays a protective role against cell damage caused by pathological factors [16]. Puerarin has been reported to reduce Caspase- 3 expression by activating PI3K/AKT signaling pathway, and significantly improve tumor necrosis factor- $\alpha$ (TNF- $\alpha$ ) induced apoptosis in PC12 cells [17]. In addition, Chen et al. showed that puerarin can directly act on myocytes, alleviate mitochondrial dysfunction, mitosis and inflammatory response induced by palmitate, thereby contributing to the improvement of insulin sensitivity and the improvement of impaired insulin signaling in skeletal muscle and isolated myotubes of diabetic animals [18]. As mentioned above, puerarin may play an active role in targeted therapy of chronic obstructive pneumonia disease.

\section{RESULTS}

\section{Puerarin promotes the proliferation and inhibits apoptosis of CSE-induced HBECs}

To further explore the effect of puerarin on the proliferation and apoptosis of CSE-induced HBECs, different concentrations of puerarin were used to intervene in model cells. MTT assay results showed that the gradual increase of puerarin concentration could significantly promote the proliferation of HBECs compared with $20 \%$ CSE treatment alone (Figure 1A). Next, the results of flow cytometry showed that the apoptosis of HBECs treated with $20 \%$ CSE was significantly increased, but this trend was reversed after puerarin intervention at different concentrations, and the inhibition of apoptosis by high concentrations of puerarin was more significant (Figure 1B). Western blotting results suggested that the expression of apoptosis-related genes, including Cleaved caspase 3 and Bax, decreased after puerarin intervention and was significantly correlated with the concentration (Figure 1C).

\section{Puerarin inhibits mitochondrial autophagy of CSE- induced HBECs}

To clarify the effect of puerarin on mitochondria, puerarin with concentrations of $50 \mu \mathrm{g} / \mathrm{ml}, 100 \mu \mathrm{g} / \mathrm{ml}$ and $200 \mu \mathrm{g} / \mathrm{ml}$ was used to interfere with $20 \%$ CSEinduced HBECs, respectively. The changes of MMP level were detected by flow cytometry after labeling with JC-1 probe and the results showed that MMP level was significantly decreased after $20 \%$ CSE treatment, suggesting early apoptosis of cells. However, the decrease of membrane potential was gradually reversed after puerarin intervention at different concentrations, and was significantly correlated with puerarin concentration (Figure 2A). In addition, the results of flow cytometry suggested that ROS levels in HBECs increased significantly after $20 \%$ CSE treatment, while puerarin intervention could down-regulate ROS levels in cells, which was significantly correlated with the concentration (Figure 2B). Mitochondria are the main site of ATP synthesis. Treating cells with $20 \%$ CSE leads to damage of mitochondrial structure and function, and ATP content in cells decreases. Puerarin at different concentrations can significantly improve mitochondrial damage and increase the amount of ATP synthesis (Figure 2C). Western blotting was used to detect the expression levels of mitochondrial autophagy-related proteins. The results showed that the protein expression of PINK1 and Parkin decreased significantly with the increase of puerarin intervention concentration compared with $20 \%$ CSE treatment alone, suggesting that puerarin had an inhibitory effect on mitochondrial autophagy (Figure 2D). 


\section{Puerarin down-regulates the expression of DRP1 and FUNDC1}

To investigate whether CSE-induced autophagy is associated with DRP1 and FUNDC1, the expression of DRP1 and FUNDC1 was measured. The results of RTqPCR and Western blotting showed that DRP1 mRNA and protein expression were significantly increased after treatment of cells with $20 \%$ CSE, but this trend was reversed with the increasing concentration of puerarin intervention (Figure 3A, 3B). Similarly, the expression of FUNDC1 mRNA and protein was significantly upregulated in 20\% CSE-treated group, but its expression was down-regulated after puerarin intervention and significantly correlated with the concentration (Figure 3C, 3D). In addition, Western blotting results showed that expression of phosphorylated FUNDC1 protein was significantly down-regulated after $20 \%$ CSE treatment, and puerarin intervention at different concentrations could significantly reverse its expression, which was correlated with the concentration. This suggests that FUNDC1 inhibits mitochondrial autophagy in the form of phosphorylation (Figure 3D).

\section{Puerarin prevents mitochondrial autophagy by inhibiting dephosphorylation of FUNDC1}

To further explore whether puerarin prevents mitochondrial autophagy by inhibiting the dephosphorylation of FUNDC1, PH0321, a protein phosphatase inhibitor, was used to inhibit the dephosphorylation of FUNDC1. Western blotting results showed that the expression of p-FUNDC1 was decreased and mitochondrial autophagy was promoted after PH0321 treatment. The expression of p-FUNDC1 was significantly increased after intervention with high concentration of puerarin, which inhibited mitochondrial autophagy (Figure 4A). Next, the results of flow cytometry suggested that PH0321 treatment alone significantly decreased MMP level, and MMP level significantly increased after high-concentration puerarin intervention (Figure 4B). In addition, the content of ROS in cells was significantly increased after PH0321 treatment, but this trend was reversed by high concentration puerarin intervention, which significantly decreased the content of ROS in cells (Figure 4C). Furthermore, the inhibitory effect of PH0321 on ATP synthesis was reversed after high-concentration puerarin intervention (Figure 4D). Western blotting results showed that the expression of mitochondrial autophagy-related proteins such as PINK1 and Parkin was significantly upregulated after PH0321 treatment, and the expression of these two proteins was significantly downregulated after highconcentration puerarin intervention (Figure 4E). The above results indicate that puerarin prevents mitochondrial autophagy by inhibiting the dephosphorylation of FUNDC1.

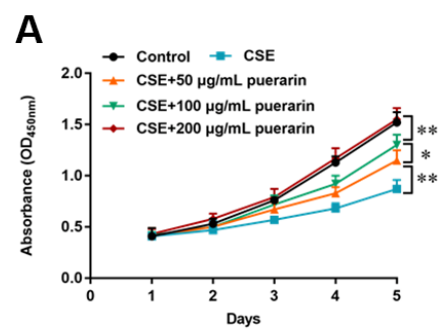

B


Figure 1. Puerarin promotes the proliferation and inhibits apoptosis of CSE-induced HBECs. HBECs was induced by $20 \%$ CSE and intervened with $50 \mu \mathrm{g} / \mathrm{mL}, 100 \mu \mathrm{g} / \mathrm{mL}$ and $200 \mu \mathrm{g} / \mathrm{mL}$ puerarin for $12 \mathrm{~h}$, respectively. (A) MTT assay was used to measure the viability of CSEinduced HBECs. (B) Apoptosis of CSE-induced HBECs were detected by flow cytometry. (C) The protein expression of Cleaved caspase3 and Bax in CSE-induced HBECs were analyzed by Western blotting. $\beta$-actin was used as an invariant internal control for calculating protein-fold changes. $\mathrm{N}=6, * P<0.05, * * P<0.01$. 


\section{Puerarin inhibits apoptosis of CSE-induced HBECs by inhibiting mitochondrial autophagy}

To further explore the effect of puerarin on apoptosis after inhibiting mitochondrial autophagy, we blocked the process of autophagy by using Mdivi, a mitochondrial division protein, as an inhibitor of mitochondrial autophagy. The results of Western blotting suggested that the expression of related proteins, such as PINK1 and Parkin, was significantly decreased after the mitochondrial autophagy process was blocked, and the changes of both proteins were consistent with the high concentration puerarin intervention (Figure 5A). Furthermore, MTT assay was used to detect cell viability and the results showed that the inhibition of $20 \%$ CSE on cell viability was reversed after treatment with mitochondrial autophagy inhibitors, which showed that the cell viability was the same as that in the high concentration puerarin intervention group (Figure 5B). Next, autophagy inhibitor treatment significantly reduced the apoptosis rate, and the expression of apoptosis- related proteins such as Cleaved caspase 3 and Bax was also significantly downregulated, which showed the same trend as the highconcentration puerarin intervention (Figure 5C, 5D).

Puerarin inhibits mitochondrial autophagy and apoptosis in CSE-induced HBECs by activating the $\mathrm{PIBK} / \mathrm{AKT} / \mathrm{mTOR}$ signaling pathway

The PI3K/AKT/mTOR signaling pathway widely exists in a variety of cells and participates in a variety of physiological and pathological processes in vivo, and its activation has an inhibitory effect on cell autophagy. To further explore whether inhibits mitochondrial autophagy and apoptosis in CSE-induced HBECs by activating the PI3K/AKT/mTOR signaling pathway, 3MA, a PI3K inhibitor, was used to block the pathway. It was found that the protein expression of $\mathrm{p}-\mathrm{PI} 3 \mathrm{~K}$, $\mathrm{p}$ AKT and p-mTOR were significantly reduced in CSEinduced HBECs, while its expression was significantly reversed after high concentration puerarin intervention,
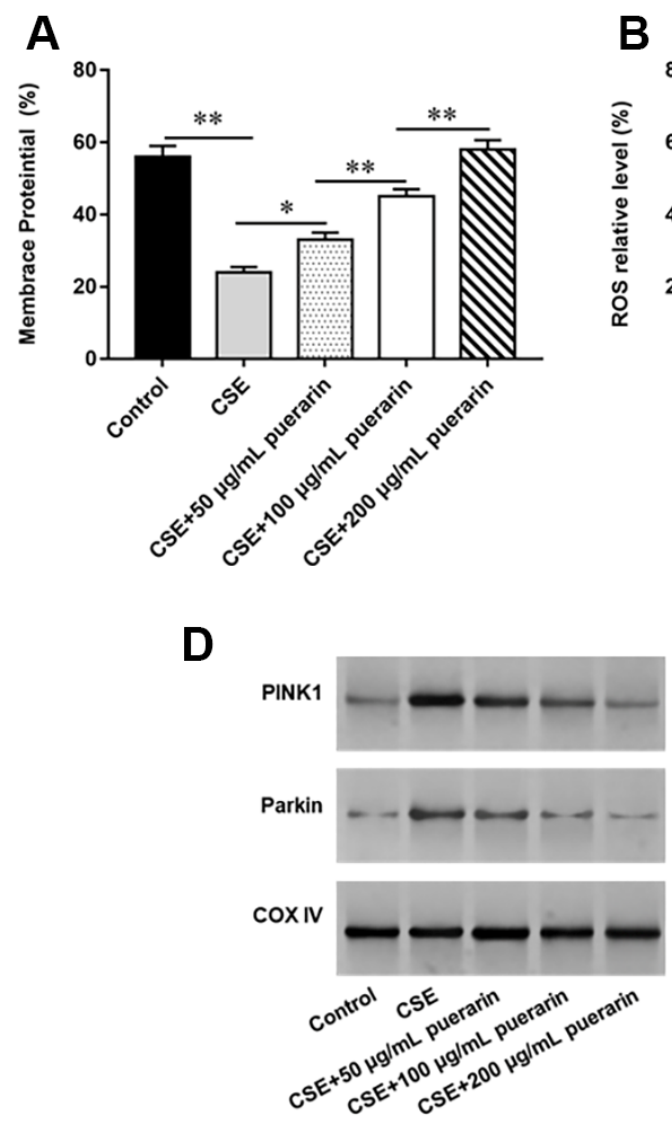

B

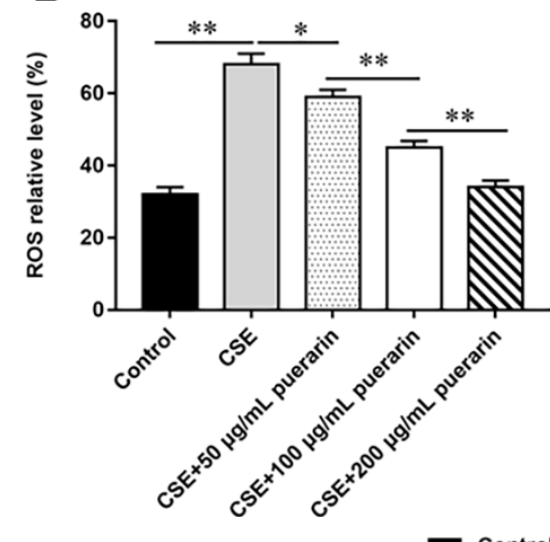

- Control $\square$ CSE

CSE $+50 \mu \mathrm{g} / \mathrm{mL}$ puerarin

$\square \mathrm{CSE}+100 \mu \mathrm{g} / \mathrm{mL}$ puerarin

$\Delta \mathrm{CSE}+200 \mu \mathrm{g} / \mathrm{mL}$ puerarin

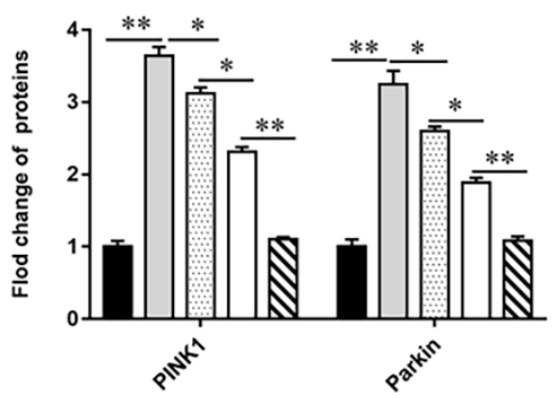

Figure 2. Puerarin inhibits mitochondrial autophagy of CSE-induced HBECs. HBECs was induced by $20 \%$ CSE and intervened with 50 $\mu \mathrm{g} / \mathrm{mL}, 100 \mu \mathrm{g} / \mathrm{mL}$ and $200 \mu \mathrm{g} / \mathrm{mL}$ puerarin for $12 \mathrm{~h}$, respectively. (A) Flow cytometry (JC-1) was used to detect changes in MMP level in CSEinduced HBECs. (B) Mitochondrial ROS levels were detected by flow cytometry (DCFH-DA). (C) The content of ATP in CSE-induced HBECS was detected with kits. (D) Western blotting was used to detect the expression of mitochondrial autophagy-related proteins such as PINK1 and Parkin. $\beta$-actin was used as the loading control. $\mathrm{N}=6,{ }^{*} P<0.05,{ }^{*} P<0.01$. 
suggesting that puerarin can activate the $\mathrm{PI}$ K $/ \mathrm{AKT} / \mathrm{mTOR}$ signaling pathway (Figure 6A). Next, we blocked PI3K with 3-MA, and as expected, the expression of $\mathrm{p}-\mathrm{PI} 3 \mathrm{~K}$, p-AKT, and p-mTOR in cells was significantly decreased after pathway blockade compared with the high-concentration puerarin-treated group, and this signaling pathway was inhibited (Figure $6 \mathrm{~B})$. Western blotting results showed that the expression of mitochondrial autophagy-related proteins such as PINK1 and Parkin increased significantly after the pathway was blocked (Figure 6C), and the expression of apoptosis-related proteins such as Cleaved Casepase3 and Bax was also significantly upregulated (Figure 6D). The above results further verified that the inhibition of puerarin on mitochondrial autophagy and apoptosis in CSE-induced HBECs was accomplished by activating the $\mathrm{PI} 3 \mathrm{~K} / \mathrm{AKT} / \mathrm{mTOR}$ signaling pathway.

\section{DISCUSSION}

Mitochondria are energy factories in eukaryotic cells, which can provide energy for various physiological activities of the body [7]. With the occurrence of mitochondrial oxidative phosphorylation, a lot of ROS is to be produced, and excessive ROS will lead to mitochondrial damage, causing apoptosis [19]. Therefore, clearance of damaged mitochondria through autophagy, a degradation pathway, is essential to maintain the stability of the intracellular environment.

FUNDC1 plays a key regulatory role in the biological process of mitochondrial autophagy. FUNDC1 is localized on the outer mitochondrial membrane and participates in mitochondrial autophagy caused by hypoxia and reduced mitochondrial membrane potential, in which the phosphorylated form of FUNDC1 inhibits autophagy while dephosphorylation promotes autophagy [20, 21]. Wang et al. found that mitochondrial DNA copy number and mitochondrial protein expression were significantly decreased in Ginkgolic acids-treated mouse bone marrow stromal cells, but FUNDC1 gene knockout restored Ginkgolic acids-induced changes in mitochondrial mass loss and mitochondrial membrane potential loss [22]. Another
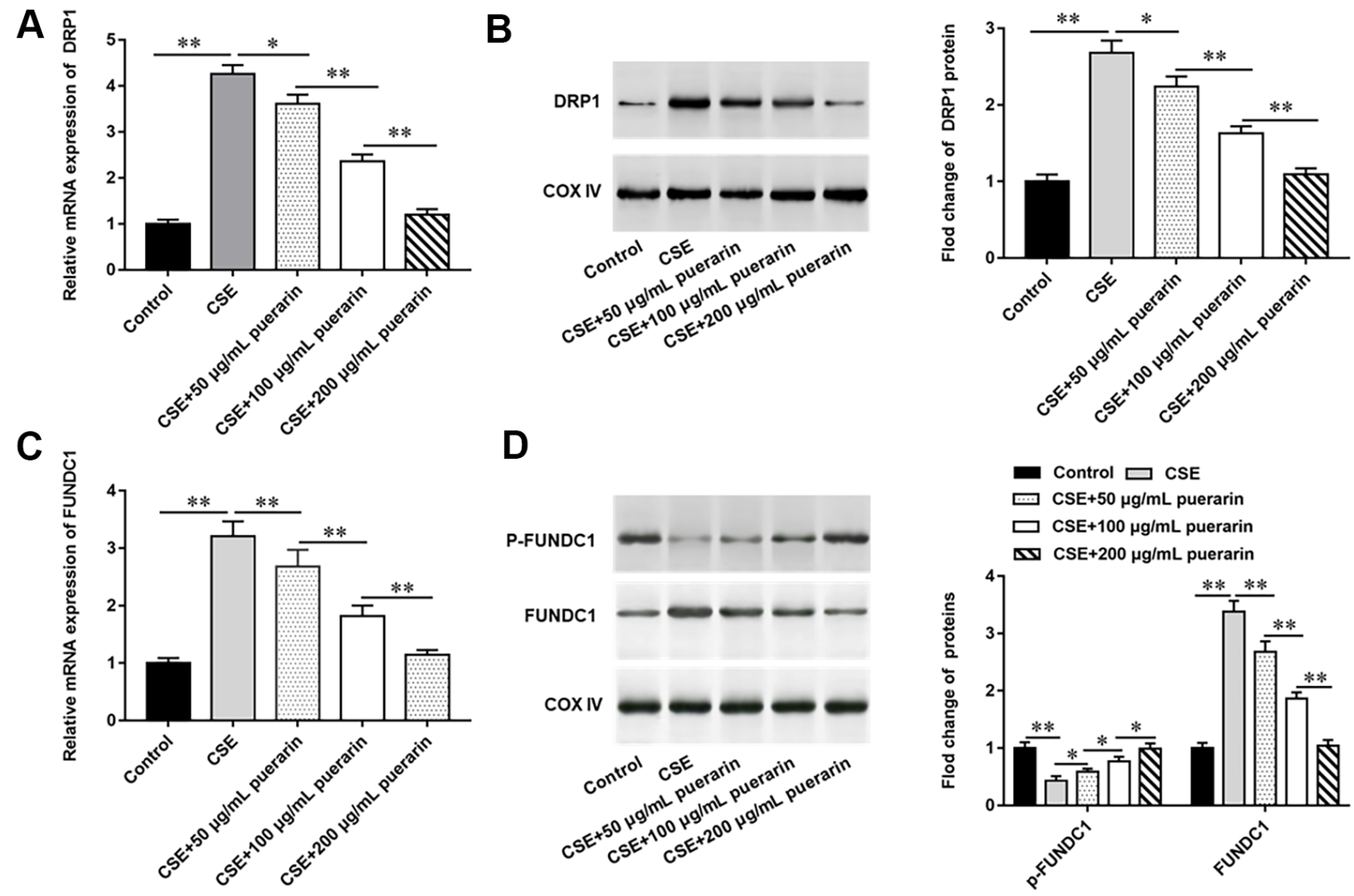

Figure 3. Puerarin down-regulates the expression of DRP1 and FUNDC1. HBECs was induced by $20 \%$ CSE and intervened with 50 $\mu \mathrm{g} / \mathrm{mL}, 100 \mu \mathrm{g} / \mathrm{mL}$ and $200 \mu \mathrm{g} / \mathrm{mL}$ puerarin for $12 \mathrm{~h}$, respectively. (A) Relative mRNA expression of DRP1 was analyzed by RT-qPCR. (B) Western blotting was used to measure the protein expression of DRP1. (C) Relative expression of FUNDC1 was detected by RT-qPCR. (D) The protein expression of FUNDC1 and p-FUNDC1 in CSE-induced HBECs were analyzed by Western blotting. $\beta$-actin was used as an internal reference. $\mathrm{N}=6, * P<0.05, * * P<0.01$. 
study showed that FUNDC1 plays an important regulatory role in hypoxia-induced $\mathrm{PC} 12$ neuroautophagy and apoptosis, and overexpression of FUNDC1 can promote autophagy and then prevent neuronal apoptosis [20]. In myocardial ischemiareperfusion injury model, FUNDC1-mediated mitochondrial autophagy was inhibited, damaged mitochondria could not be cleared in time and accumulated in a large amount in cells, leading to microvascular endothelial cell apoptosis and barrier dysfunction [23]. In addition, some studies have found that hydrogen peroxide can up-regulate the expression of FUNDC1 by activating the ERK1/2 signaling, triggering mitochondrial autophagy, making laryngeal cancer cells more suitable for survival [24].

In recent years, more and more studies have found that mitochondrial autophagy plays an important regulatory role in the pathogenesis of COPD. Among them, Araya Jun et al. found that CS-exposed mouse airway epithelial cells showed impaired mitochondrial volume accumulation, increased oxidative modification and accelerated cell senescence, and experimental animals showed enhanced airway wall thickening and emphysema changes [25]. The upstream regulatory mechanism of mitochondrial autophagy is still unclear, and it is generally accepted that mitochondrial division is one of the important upstream regulatory factors of mitochondrial autophagy. Among them, DRP1 is a key executor. Normally, mitochondria maintain cell energy supply by controlling their number through division, but excessive mitochondrial division leads to the production of a large number of dysfunctional mitochondrial fragments, which in turn triggers mitochondrial autophagy [26-28]. In this study, we found that CSEinduced HEBCs could significantly upregulate the expression of DRP1 and FUNDC1, as well as the expression of autophagy-related proteins and apoptosisrelated proteins, indicating that mitochondrial autophagy plays a positive regulatory role on apoptosis.
A

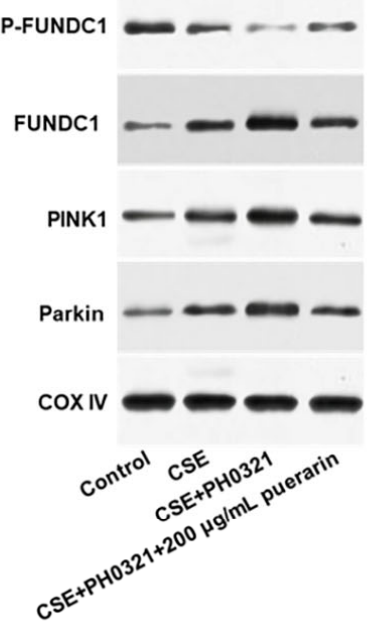

C

- Control 四 CSE $\square$ CSE+PH0321 $\triangle \mathrm{CSE}+\mathrm{PH} 0321+200 \mu \mathrm{g} / \mathrm{mL}$ puerarin

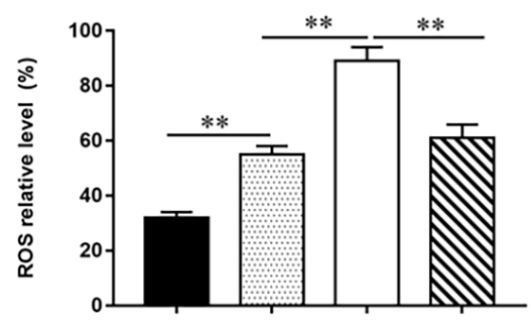

Control $\square$ CSE $\square$ CSE+PH0321 $\triangle \mathrm{CSE}+\mathrm{PH} 0321+200 \mu \mathrm{g} / \mathrm{mL}$ puerarin

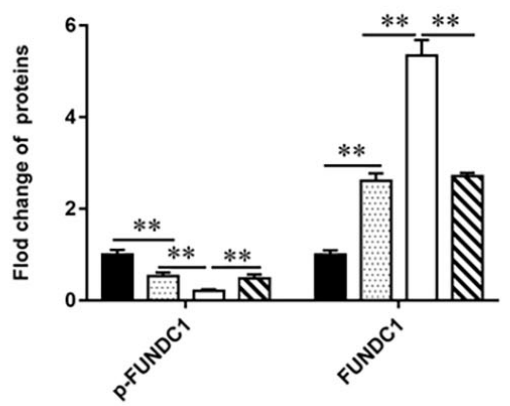

D


B

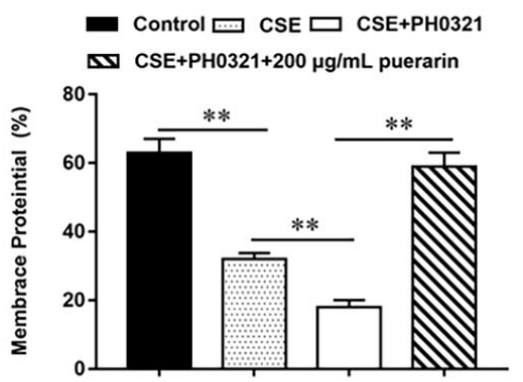

E
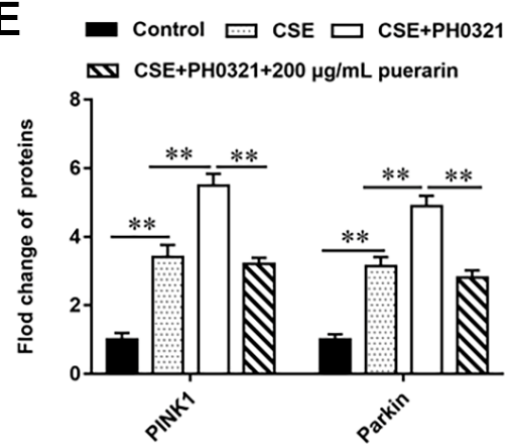

Figure 4. Puerarin prevents mitochondrial autophagy by inhibiting dephosphorylation of FUNDC1. PH0321, a protein phosphatase inhibitor, treated $20 \% \mathrm{CSE}$-induced HBECs and then co-cultured with puerarin at the concentration of $200 \mu \mathrm{g} / \mathrm{mL}$. (A) The protein expression of FUNDC1 and p-FUNDC1 in CSE-induced HBECs were analyzed by Western blotting. (B) Flow cytometry (JC-1) was used to detect changes in mitochondrial membrane potential in CSE-induced HBECs. (C) Mitochondrial ROS levels were detected by flow cytometry (DCFH-DA). (D) The content of ATP in CSE-induced HBECs was detected with kits. (E) Western blotting was used to detect the expression of mitochondrial autophagy-related proteins such as PINK1 and Parkin. $\beta$-actin was used as an invariant internal control for calculating protein-fold changes. $\mathrm{N}=6, * * P<0.01$. 
At present, there are few studies on the regulatory relationship between puerarin and DRP1 or FUNDC1, but the effect of flavonoids on mitochondrial autophagy through regulating DRP1 has been widely reported. Isoliglycyrrhizin isolated from Licorice attenuates neuronal cell death mediated by loss of mitochondrial membrane potential by promoting dephosphorylation of DRP1, inhibiting ROS generation and calcium ion levels in cells, and preventing glutamate-induced mitochondrial fission [29]. In a mouse model of Alzheimer's disease, icariin treatment led to a decrease in DRP1, promoted mitochondrial transport, and protected mitochondria from fragmentation, suggesting that it may be a potential therapeutic supplement for neurodegenerative diseases associated with Alzheimer's disease and other mitochondrial dysfunctions [30].
A

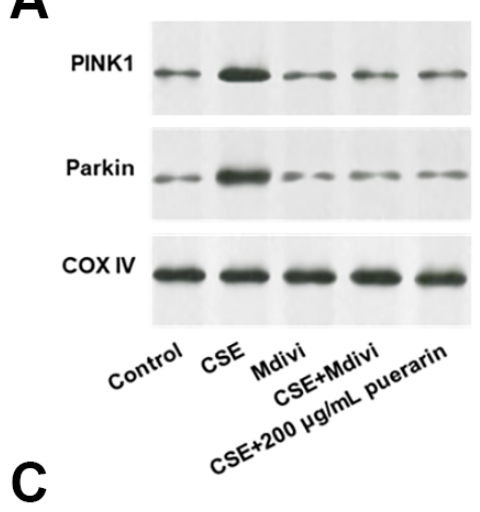

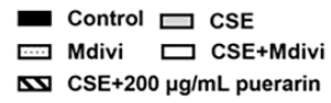

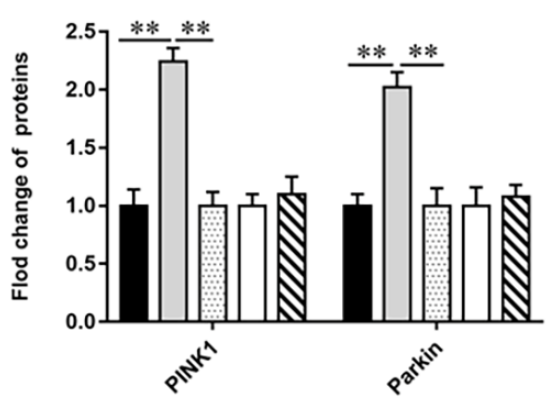

B

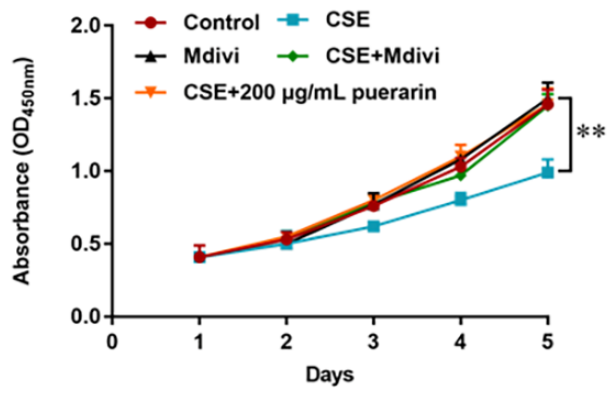

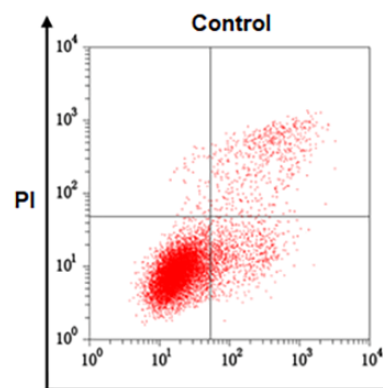


$\mathrm{CSE}+200 \mu \mathrm{g} / \mathrm{mL}$ puerarin

Annexin VFITC

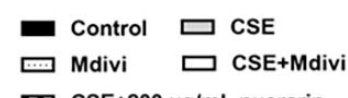

$\Delta \mathrm{CSE}+200 \mu \mathrm{g} / \mathrm{mL}$ puerarin

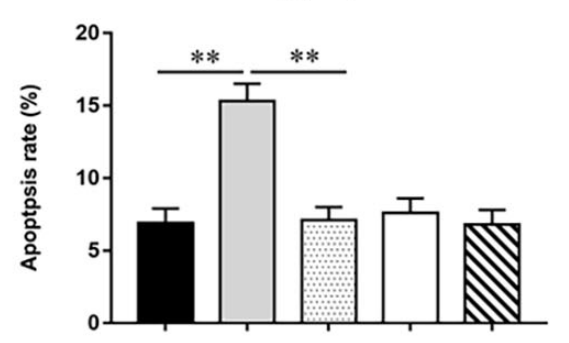

D

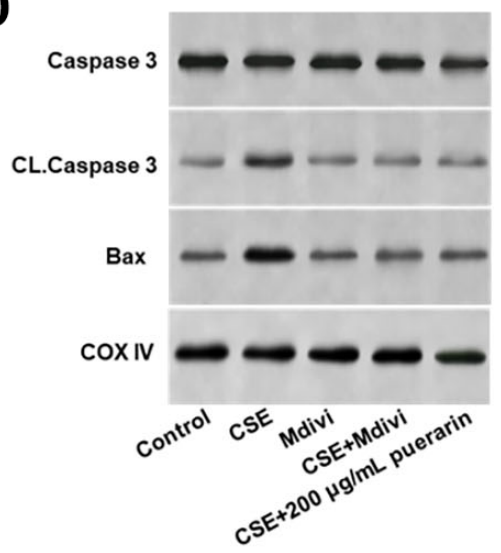

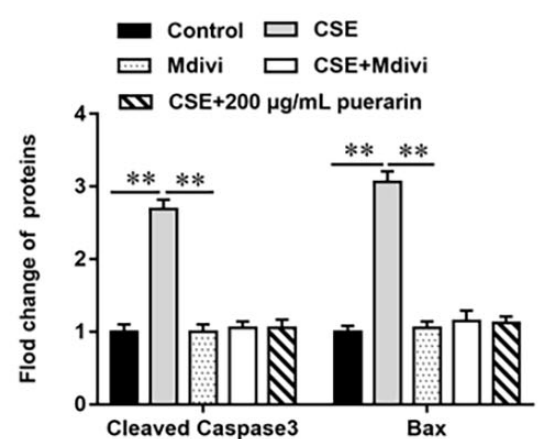

Figure 5. Puerarin inhibits apoptosis of CSE-induced HBECs by inhibiting mitochondrial autophagy. Mdivi, a mitochondrial autophagy inhibitor, treated $20 \%$ CSE-induced HBECs and then co-cultured with puerarin at a concentration of $200 \mu \mathrm{g} / \mathrm{mL}$. (A) Western blotting was used to detect the expression of mitochondrial autophagy-related proteins such as PINK1 and Parkin. (B) MTT assay was used to measure the viability of CSE-induced HBECs. (C) Apoptosis of CSE-induced HBECs were detected by flow cytometry. (D) The protein expression of Cleaved caspase3 and Bax in CSE-induced HBECs were analyzed by Western blotting. $\beta$-actin was used as the loading control. $\mathrm{N}=6,{ }^{* *} P<0.01$. 
Other studies have reported that kaempferol protects neurons from succinate-mediated ischemic injury by inhibiting mitochondrial division by activating the AKT axis [31].

We found that different concentrations of puerarin could significantly reverse the damage of CES on HEBCs, which was manifested by restoring MMP level, reducing ROS generation, promoting ATP synthesis, reducing mitochondrial autophagy, and inhibiting apoptosis. We further found that puerarin inhibited mitochondrial autophagy and mitigated cell injury by inhibiting the expression of DRP1 and FUNDC1, and this protective effect may be closely related to the activation of $\mathrm{PI} 3 \mathrm{~K} / \mathrm{AKT} / \mathrm{mTOR}$ signaling pathway. Puerarin has been reported to alleviate oxidative stress by inhibiting lead-induced renal cell apoptosis, restoring the balance between Bax and Bcl-2, and reducing mitochondrial Cyt $\mathrm{C}$ release through activating the PI3K/AKT/eNOS pathway [32]. Puerarin can attenuate mitochondrial ultrastructure destruction, change MMP level to reverse cadmium-induced ATP depletion, and increase $\mathrm{Bcl}-2 / \mathrm{Bax}$ ratio, blocking Casepase 3 activation [33]. A recent study claimed that the tuber extract of Alisma orientale alleviated airway inflammation and emphysema phenotype in mice by activating mTOR and inhibiting autophagy [34].

In conclusion, our study shows that puerarin can inhibit FUNDC1-mediated mitochondrial autophagy and bronchial epithelial cell apoptosis by activating the $\mathrm{PI} 3 \mathrm{~K} / \mathrm{AKT} / \mathrm{mTOR}$ signaling pathway to achieve cytoprotective effects, which provides a new idea for the treatment of COPD.

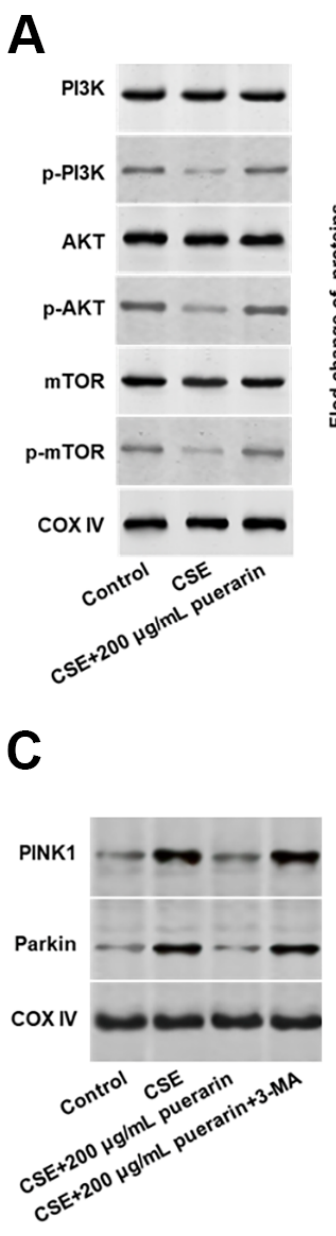

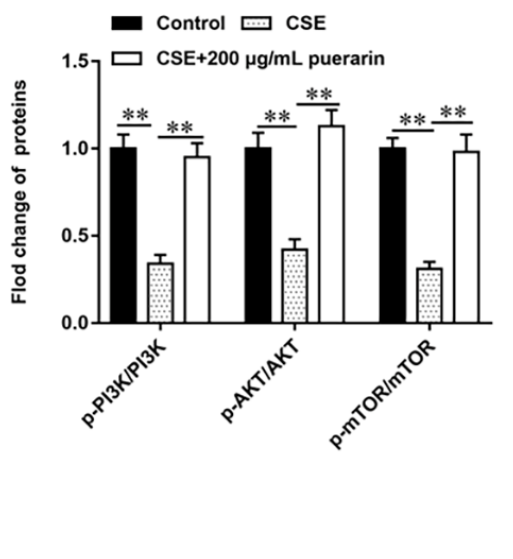
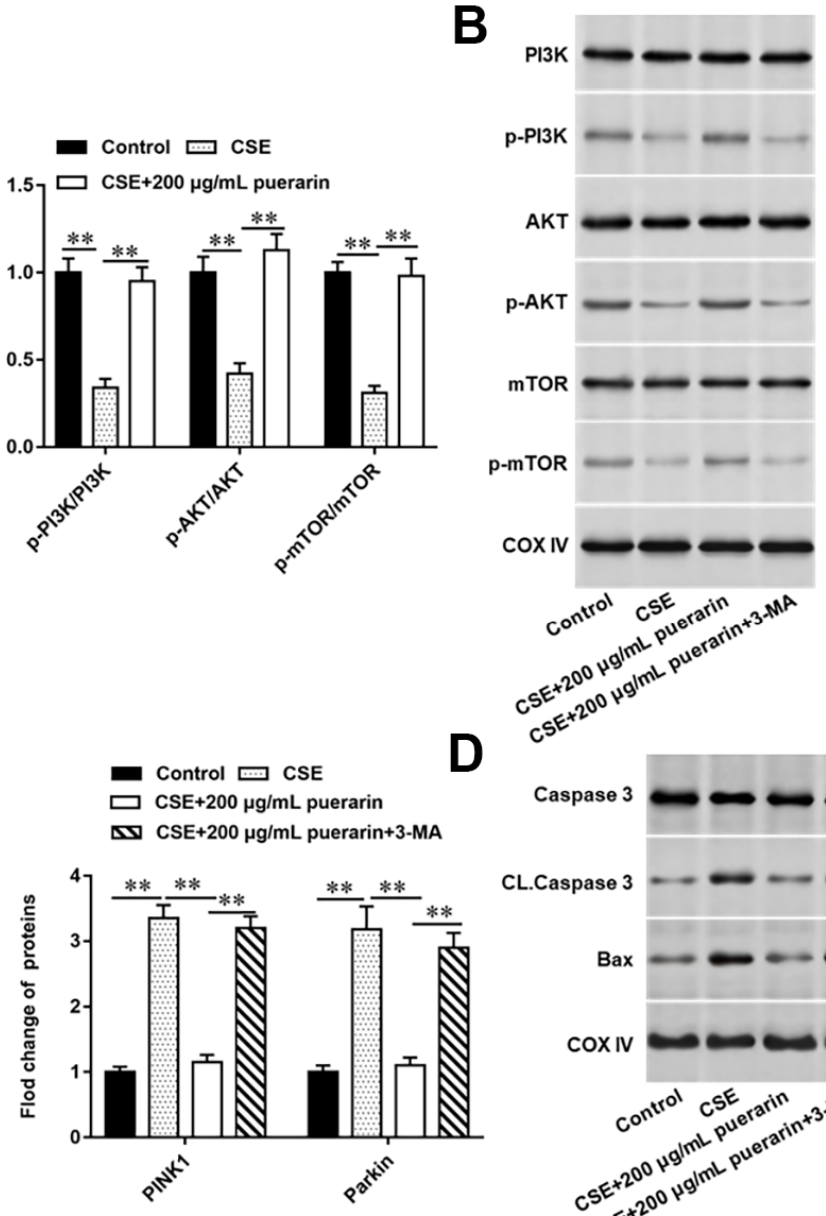
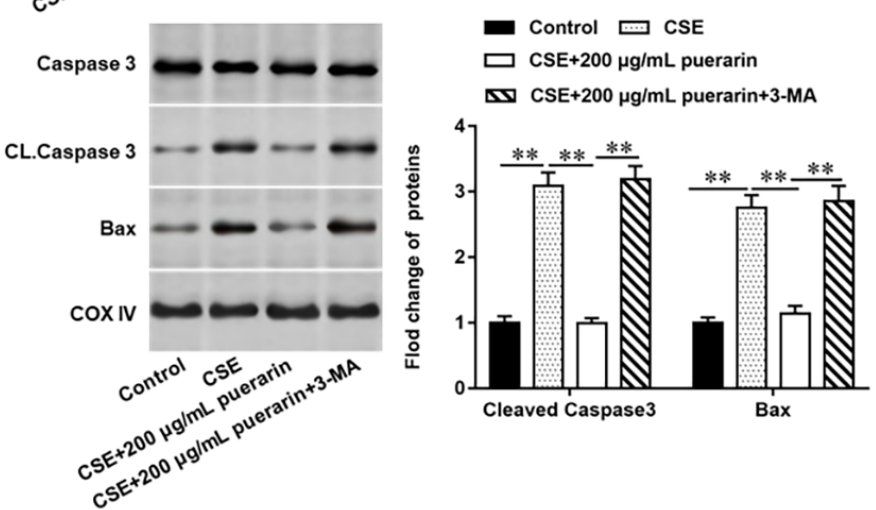

Figure 6. Puerarin inhibits mitochondrial autophagy and apoptosis in CSE-induced HBECs by activating the PI3K/AKT/mTOR signaling pathway. 3-MA, an inhibitor of PI3K, was added to $20 \%$ CES-induced HEBCs, which treated with puerarin at a concentration of $200 \mu \mathrm{g} / \mathrm{mL}$. (A, B) The protein expression of total PI3K, p-PI3K, total AKT, p-AKT, total mTOR and p-mTOR in CSE-induced HBECs were analyzed by Western blotting. (C) Western blotting was used to detect the expression of mitochondrial autophagy-related proteins such as PINK1 and Parkin. (D) The protein expression of Cleaved caspase3 and Bax in CSE-induced HBECs were analyzed by Western blotting. $\beta$-actin was used as the loading control. $\mathrm{N}=6, * * P<0.01$. 


\section{MATERIALS AND METHODS}

\section{Cell culture}

The HBECs were purchased from the American Type Culture Collection (ATCC; https://www.atcc.org/) and stored in Dulbecco's modified Eagle's medium (DMEM, Sigma-Aldrich Chemical Company, St Louis, MO, USA) containing 10\% FBS (Sigma-Aldrich Chemical Company, St Louis, MO, USA) and penicillinstreptomycin solution (100×; Thermo Fisher Scientific Inc., Waltham, MA, USA). Puerarin was provided by Shanghai McLean Biochemical Technology Company, batch number: C11030370, purity $>98 \%$. The suspension was prepared with sterilized saline at the concentration of $50 \mu \mathrm{g} / \mathrm{mL}, 100 \mu \mathrm{g} / \mathrm{mL}$ and $200 \mu \mathrm{g} / \mathrm{mL}$ and stored in a refrigerator at $4^{\circ} \mathrm{C}$ in the dark for subsequent experiments.

\section{Preparation of $100 \%$ cigarette smoke extract}

The cigarettes used in this experiment were all Nanjing brand cigarettes. The cigarette collection system was assembled according to the cigarette support tube, gas collection bottle, buffer bottle and peristaltic pump. During gas collection, after adding about $10 \mathrm{~mL}$ DMEM culture medium into the gas collection bottle, start the peristaltic pump, adjust the speed about 160 $\mathrm{r} / \mathrm{min}$, and the gas flow rate is about $600 \mathrm{~mL} / \mathrm{min}$, ignite 1.5 cigarettes in turn, and shake the gas collection bottle continuously to dissolve the cigarette smoke as soon as possible. After the cigarette is burned out, the liquid obtained is recorded as $100 \%$ CSE, placed on the ultraclean table for filtration and sterilization. After that, the CSE solution was added into DMEM to prepare the CSE mixture, which held a concentration of $20 \%$ and used as soon as possible within $30 \mathrm{~min}$.

\section{RNA extraction and RT-qPCR}

Total RNA was isolated from HEBCs by using the TRIzol (Invitrogen, Carlsbad, CA, USA). Single-stranded cDNA was synthesized with the PrimeScript Reagent Kit (Promega, USA). Real-time PCR was conducted by using SYBR Premix Ex TaqTM Kit (Applied Biosystems, Foster City, CA, USA). The reaction was run in ABI7500 Real-time PCR system (Applied Biosystems, Carlsbad, CA, USA). GAPDH was used as an endogenous control. Briefly, $2 \mu \mathrm{L}$ of cDNA was added to $10 \mu \mathrm{L}$ of the $1 \times$ SYBR green PCR master mix with $0.4 \mu \mathrm{L}$ of Taq polymerase enzyme (RiboBio, China), $0.8 \mu \mathrm{L}$ of each primer and $6 \mu \mathrm{L} \mathrm{ddH}_{2} \mathrm{O}$ to a final volume of $20 \mu \mathrm{L}$. The RT-qPCR cycling conditions consisted of: $95^{\circ} \mathrm{C}$ for 3 $\mathrm{min}$; then 35 cycle amplification for $20 \mathrm{~s}$ at $95^{\circ} \mathrm{C}, 30 \mathrm{~s}$ at $55^{\circ} \mathrm{C}, 15 \mathrm{~s}$ at $72^{\circ} \mathrm{C}$; followed by $1 \mathrm{~min}$ at $72^{\circ} \mathrm{C}$. The primers used in this study were synthesized from Sangon
Biotech (Shanghai, China). The levels of mRNA were normalized by using the $2^{-\Delta \Delta \mathrm{Ct}}$ method.

\section{Western blot analysis}

The cells were lysed for $20 \mathrm{~min}$ on ice in ice-cold lysis buffer (Roche). The lysates were centrifuged at $12,000 \times \mathrm{g}$ for $20 \mathrm{~min}$ at $4^{\circ} \mathrm{C}$ to obtain a clear lysate. The protein content of each sample was determined by using the BCA Protein Assay Kit (Thermo Scientific). Then, equal amounts of proteins $(15 \mu \mathrm{g} /$ lane $)$ were separated on a $12 \%$ sodium dodecyl sulfate polyacrylamide gel electrophoresis (SDS-PAGE) and transferred to polyvinylidenedifluoride (PVDF) membranes (Bio-Rad, Hercules, CA, USA). The membranes were blocked in $5 \%(\mathrm{w} / \mathrm{v})$ nonfat dry milk in TBST (Tris-buffered saline$0.1 \%$ Tween) at $25^{\circ} \mathrm{C}$ for $3 \mathrm{~h}$ and then incubated with the following primary antibodies: rabbit monoclonal anti- $\beta$ actin antibody (1:900, Abcam, ab179467), rabbit monoclonal anti-PINK1 antibody (1:1000, Abcam, ab216144), mouse monoclonal anti-parkin antibody (1:1500, Abcam, ab77924), rabbit polyclonal antiCleaved casepase3 antibody (1:1100, Abcam, ab2302), rabbit monoclonal anti-Bax antibody (1:2000, Abcam, ab32503), rabbit monoclonal anti-DRP1 antibody (1:1000, Abcam, ab184247), rabbit polyclonal antiFUNDC1 antibody (1:500, Abcam, ab224722), rabbit monoclonal anti-PI3K antibody (1:1000, Abcam, ab32089), rabbit polyclonal anti-AKT antibody (1:1500, Abcam, ab179463), rabbit polyclonal anti-mTOR antibody (1:2000, Abcam, ab2732). The bands were incubated with horseradish peroxidase (HRP) conjugated goat anti-rabbit IgG (1:15000, Boster) for $1 \mathrm{~h}$ at room temperature. The bands were visualized by using an ECL Plus Chemiluminescence Reagent Kit (Pierce, Rockford, IL, USA) and were photographed by a chemiluminescence imaging system. Image $J$ software was used to quantify the band densities.

\section{Detection of MMP level by flow cytometry}

Cells were collected by $0.25 \%$ trypsin (containing EDTA) digestion method; 200 mesh screen filtration, cell counting, $1.5 \times 10^{5}$ cells were collected; cells were resuspended by adding $1 \mathrm{~mL}$ JC-1 working solution (1×) under dark conditions, incubated at $37^{\circ} \mathrm{C}$ for $20 \mathrm{~min}$ in dark, shaken once at 2 3 min intervals; and centrifuged at $4^{\circ} \mathrm{C}$ for $1500 \mathrm{rmp}$; the hearts were washed twice with JC-1 staining buffer $(1 \times)$ for $5 \mathrm{~min}$, and the cells were resuspended with $300 \mu \mathrm{L} \mathrm{JC}-1$ staining buffer $(1 \times)$ for flow cytometry detection.

\section{Detection of ROS content by flow cytometry}

Cells were collected by $0.25 \%$ trypsin (containing EDTA) digestion method; cells were filtered through 
200 mesh screen, counted, and $1.5 \times 10^{5}$ cells were collected; preparation of DCFH-DA working solution: incomplete cell culture solution is 1:1000; cells were resuspended by adding DCFH-DA working solution under dark conditions, and incubated at 37 degrees in the dark for $20 \mathrm{~min}$; cells were washed three times with incomplete cell culture medium and resuspended with $300 \mu \mathrm{L}$ of incomplete cell culture medium for flow cytometry detection.

\section{ATP content detection}

Cells were collected by digestion with $0.25 \%$ trypsin (including EDTA); ATP lysate was added, mixed with shaking, ice bath for $10 \mathrm{~min}, 4^{\circ} \mathrm{C}$ freezing centrifuge for $12000 \times \mathrm{g}$ Centrifuge for $5 \mathrm{~min}$, extract the supernatant; prepare ATP detection working solution with ATP detection reagent: ATP detection diluent $=1: 4$ in dark condition; $100 \mu \mathrm{L}$ working fluid was added into 96-well plate, stand at room temperature for 2 5 minutes, then add standard diluent and sample $20 \mu \mathrm{L}$, and quickly mixed with a pipette gun; RUL was measured on a microplate reader and ATP concentration was calculated; protein concentration was measured at the same time, and the ratio of ATP concentration: protein concentration was shown as the final result $(\mathrm{nmoL} / \mathrm{mg})$.

\section{Statistical analysis}

All statistical analyses were performed by using the SPSS software (ver. 22.0; SPSS, Chicago, IL, USA). The quantitative data derived from three independent experiments are expressed as mean \pm SEM. Significance was determined by one-way ANOVA or $t$ test. Values of $P<0.05$ were considered statistically significant.

\section{AUTHOR CONTRIBUTIONS}

WW designed and supervised the study. LW conducted the experiments and drafted the manuscript. YX collected and analyzed the data. JW contributed the methodology and analyzed the data. WJ edited the manuscript. All authors read and approved the final manuscript.

\section{CONFLICTS OF INTEREST}

The authors declare that they have no conflicts of interest.

\section{FUNDING}

This study was supported by the Medical Science and Technology Program of Zhejiang Province in China (2021KY226).

\section{REFERENCES}

1. Zhai PY, Li HX, Zhao RF, Li XQ, Wang HQ. [Clinical characteristics of pneumoconiosis complicated with chronic obstructive pulmonary disease]. Zhonghua Lao Dong Wei Sheng Zhi Ye Bing Za Zhi. 2019; 37:899-902.

https://doi.org/10.3760/cma.j.issn.1001-9391.2019. $\underline{12.005}$ PMID: $\underline{31937028}$

2. Ren $Y$, Zhang $Y$, Fan $L$, Jiao $Q$, Wang $Y$, Wang $Q$. The cullin4A is up-regulated in chronic obstructive pulmonary disease patient and contributes to epithelial-mesenchymal transition in small airway epithelium. Respir Res. 2019; 20:84. https://doi.org/10.1186/s12931-019-1048-4 PMID:31060565

3. Tang K, Zhao J, Xie J, Wang J. Decreased miR-29b expression is associated with airway inflammation in chronic obstructive pulmonary disease. Am J Physiol Lung Cell Mol Physiol. 2019; 316:L621-29. https://doi.org/10.1152/ajplung.00436.2018 PMID: $\underline{0652495}$

4. Sugiyama Y, Asai K, Yamada K, Kureya Y, ljiri N, Watanabe T, Kanazawa H, Hirata K. Decreased levels of irisin, a skeletal muscle cell-derived myokine, are related to emphysema associated with chronic obstructive pulmonary disease. Int J Chron Obstruct Pulmon Dis. 2017; 12:765-72. https://doi.org/10.2147/COPD.S126233 PMID:28424548

5. Chen $X$, Li $Y$, Hua C, Jia $P$, Xing $Y$, Xue $B$, Tian $X$, Yang $Y$, Zhang J, Qiao L, Liu H, Li X, Xie F. Establishment of rapid risk assessment model for cigarette smoke extract exposure in chronic obstructive pulmonary disease. Toxicol Lett. 2019; 316:10-19. https://doi.org/10.1016/i.toxlet.2019.08.020 PMID:31476341

6. Pouwels SD, Hesse L, Faiz A, Lubbers J, Bodha PK, Ten Hacken NH, van Oosterhout AJ, Nawijn MC, Heijink IH. Susceptibility for cigarette smoke-induced DAMP release and DAMP-induced inflammation in COPD. Am J Physiol Lung Cell Mol Physiol. 2016; 311:L881-92. https://doi.org/10.1152/ajplung.00135.2016 PMID:27612964

7. Jiang $Y$, Wang $X$, Hu D. Mitochondrial alterations during oxidative stress in chronic obstructive pulmonary disease. Int J Chron Obstruct Pulmon Dis. 2017; 12:1153-62.

https://doi.org/10.2147/COPD.S130168 PMID:28458526

8. Ryter SW, Rosas IO, Owen CA, Martinez FJ, Choi ME, Lee CG, Elias JA, Choi AM. Mitochondrial Dysfunction as a Pathogenic Mediator of Chronic Obstructive 
Pulmonary Disease and Idiopathic Pulmonary Fibrosis. Ann Am Thorac Soc. 2018 (Suppl 4); 15:S266-72. https://doi.org/10.1513/AnnalsATS.201808-585MG PMID:30759019

9. Yao RQ, Ren C, Xia ZF, Yao YM. Organelle-specific autophagy in inflammatory diseases: a potential therapeutic target underlying the quality control of multiple organelles. Autophagy. 2021; 17:385-401. https://doi.org/10.1080/15548627.2020.1725377 PMID: $\underline{32048886}$

10. Wen W, Yu G, Liu W, Gu L, Chu J, Zhou X, Liu Y, Lai G. Silencing FUNDC1 alleviates chronic obstructive pulmonary disease by inhibiting mitochondrial autophagy and bronchial epithelium cell apoptosis under hypoxic environment. J Cell Biochem. 2019; 120:17602-15.

https://doi.org/10.1002/jcb.29028

PMID:31237014

11. Lu MC, Yang MD, Li PC, Fang HY, Huang HY, Chan YC, Bau DT. Effect of Oligomeric Proanthocyanidin on the Antioxidant Status and Lung Function of Patients with Chronic Obstructive Pulmonary Disease. In Vivo. 2018; 32:753-58.

https://doi.org/10.21873/invivo.11304

PMID:29936455

12. Mitani A, Azam A, Vuppusetty C, Ito K, Mercado N, Barnes PJ. Quercetin restores corticosteroid sensitivity in cells from patients with chronic obstructive pulmonary disease. Exp Lung Res. 2017; 43:417-25. https://doi.org/10.1080/01902148.2017.1393707 PMID:29227717

13. Liu XJ, Bao HR, Zeng XL, Wei JM. Effects of resveratrol and genistein on nuclear factor-KB, tumor necrosis factor- $\alpha$ and matrix metalloproteinase- 9 in patients with chronic obstructive pulmonary disease. Mol Med Rep. 2016; 13:4266-72.

https://doi.org/10.3892/mmr.2016.5057

PMID:27035424

14. Gao J, Deng Y, Yin C, Liu Y, Zhang W, Shi J, Gong Q. Icariside II, a novel phosphodiesterase 5 inhibitor, protects against $\mathrm{H}_{2} \mathrm{O}_{2}$-induced PC12 cells death by inhibiting mitochondria-mediated autophagy. J Cell Mol Med. 2017; 21:375-86. https://doi.org/10.1111/jcmm.12971 PMID:27642051

15. Bacanlı M, Başaran AA, Başaran N. The antioxidant, cytotoxic, and antigenotoxic effects of galangin, puerarin, and ursolic acid in mammalian cells. Drug Chem Toxicol. 2017; 40:256-62.

https://doi.org/10.1080/01480545.2016.1209680 PMID:27461151

16. Xie $N$, Wang $C$, Lian $Y$, Wu C, Zhang $H$, Zhang $Q$. Puerarin protects hippocampal neurons against cell death in pilocarpine-induced seizures through antioxidant and anti-apoptotic mechanisms. Cell Mol Neurobiol. 2014; 34:1175-82.

https://doi.org/10.1007/s10571-014-0093-2

PMID:25151533

17. Liang $F$, Xie S. Puerarin prevents tumor necrosis factor$\alpha$-induced apoptosis of PC12 cells via activation of the PI3K/Akt signaling pathway. Exp Ther Med. 2017; 14:813-18.

https://doi.org/10.3892/etm.2017.4545

PMID:28673004

18. Chen $X, Y i$, Song $S$, Wang $L$, Liang $Q$, Wang $Y$, Wu $Y$, Gao Q. Puerarin attenuates palmitate-induced mitochondrial dysfunction, impaired mitophagy and inflammation in L6 myotubes. Life Sci. 2018; 206:84-92.

https://doi.org/10.1016/j.Ifs.2018.05.041 PMID:29802940

19. Li X, Yang H, Sun H, Lu R, Zhang C, Gao N, Meng Q, Wu S, Wang S, Aschner M, Wu J, Tang B, Gu A, et al. Taurine ameliorates particulate matter-induced emphysema by switching on mitochondrial NADH dehydrogenase genes. Proc Natl Acad Sci USA. 2017; 114:E9655-64.

https://doi.org/10.1073/pnas.1712465114 PMID:29078374

20. Wang L, Wang $P$, Dong $H$, Wang $S$, Chu $H$, Yan $W$, Zhang X. Ulk1/FUNDC1 Prevents Nerve Cells from Hypoxia-Induced Apoptosis by Promoting Cell Autophagy. Neurochem Res. 2018; 43:1539-48. https://doi.org/10.1007/s11064-018-2568-x PMID:29923038

21. Wu W, Li W, Chen H, Jiang L, Zhu R, Feng D. FUNDC1 is a novel mitochondrial-associated-membrane (MAM) protein required for hypoxia-induced mitochondrial fission and mitophagy. Autophagy. 2016; 12:1675-76. https://doi.org/10.1080/15548627.2016.1193656 PMID:27314574

22. Wang $W$, Wang $M$, Ruan $Y$, Tan J, Wang $H$, Yang $T$, Li J, Zhou Q. Ginkgolic Acids Impair Mitochondrial Function by Decreasing Mitochondrial Biogenesis and Promoting FUNDC1-Dependent Mitophagy. J Agric Food Chem. 2019; 67:10097-106. https://doi.org/10.1021/acs.jafc.9b04178 PMID:31418272

23. Zhou H, Wang J, Zhu P, Zhu H, Toan S, Hu S, Ren J, Chen Y. NR4A1 aggravates the cardiac microvascular ischemia reperfusion injury through suppressing FUNDC1-mediated mitophagy and promoting Mffrequired mitochondrial fission by CK2 $\alpha$. Basic Res Cardiol. 2018; 113:23.

https://doi.org/10.1007/s00395-018-0682-1

PMID:29744594 
24. Hui L, Wu H, Wang TW, Yang N, Guo X, Jang XJ. Hydrogen peroxide-induced mitophagy contributes to laryngeal cancer cells survival via the upregulation of FUNDC1. Clin Transl Oncol. 2019; 21:596-606. https://doi.org/10.1007/s12094-018-1958-5 PMID:30284230

25. Araya J, Tsubouchi $K$, Sato N, Ito $S$, Minagawa $S$, Hara $\mathrm{H}$, Hosaka Y, Ichikawa A, Saito N, Kadota T, Yoshida M, Fujita $\mathrm{Y}$, Utsumi $\mathrm{H}$, et al. PRKN-regulated mitophagy and cellular senescence during COPD pathogenesis. Autophagy. 2019; 15:510-26. https://doi.org/10.1080/15548627.2018.1532259 PMID:30290714

26. Wang J, Zhu P, Li R, Ren J, Zhou H. Fundc1-dependent mitophagy is obligatory to ischemic preconditioningconferred renoprotection in ischemic AKI via suppression of Drp1-mediated mitochondrial fission. Redox Biol. 2020; 30:101415.

https://doi.org/10.1016/j.redox.2019.101415 PMID:31901590

27. Wu W, Lin C, Wu K, Jiang L, Wang $X$, Li W, Zhuang $H$, Zhang $X$, Chen H, Li S, Yang Y, Lu Y, Wang J, et al. FUNDC1 regulates mitochondrial dynamics at the ERmitochondrial contact site under hypoxic conditions. EMBO J. 2016; 35:1368-84.

https://doi.org/10.15252/embj.201593102

PMID:27145933

28. Chen M, Chen Z, Wang Y, Tan Z, Zhu C, Li Y, Han Z, Chen L, Gao R, Liu L, Chen Q. Mitophagy receptor FUNDC1 regulates mitochondrial dynamics and mitophagy. Autophagy. 2016; 12:689-702. https://doi.org/10.1080/15548627.2016.1151580 PMID:27050458

29. Lee DG, Min JS, Lee HS, Lee DS. Isoliquiritigenin attenuates glutamate-induced mitochondrial fission via calcineurin-mediated Drp1 dephosphorylation in HT22 hippocampal neuron cells. Neurotoxicology. 2018; 68:133-41. https://doi.org/10.1016/j.neuro.2018.07.011 PMID:30048666

30. Chen Y, Han S, Huang X, Ni J, He X. The Protective Effect of Icariin on Mitochondrial Transport and Distribution in Primary Hippocampal Neurons from 3x Tg-AD Mice. Int J Mol Sci. 2016; 17:163. https://doi.org/10.3390/ijms17020163 PMID:26828481

31. Wu B, Luo H, Zhou X, Cheng CY, Lin L, Liu BL, Liu K, Li P, Yang $H$. Succinate-induced neuronal mitochondrial fission and hexokinase II malfunction in ischemic stroke: Therapeutical effects of kaempferol. Biochim Biophys Acta Mol Basis Dis. 2017; 1863:2307-18. https://doi.org/10.1016/j.bbadis.2017.06.011 PMID:28634116

32. Liu CM, Ma JQ, Sun YZ. Puerarin protects rat kidney from lead-induced apoptosis by modulating the PI3K/Akt/eNOS pathway. Toxicol Appl Pharmacol. 2012; 258:330-42. https://doi.org/10.1016/i.taap.2011.11.015 PMID:22172631

33. Song $X B$, Liu G, Wang ZY, Wang L. Puerarin protects against cadmium-induced proximal tubular cell apoptosis by restoring mitochondrial function. Chem Biol Interact. 2016; 260:219-31. https://doi.org/10.1016/i.cbi.2016.10.006 PMID:27717697

34. Kao ST, Wang SD, Lin CC, Lin L. Jin Gui Shen Qi Wan, a traditional Chinese medicine, alleviated allergic airway hypersensitivity and inflammatory cell infiltration in a chronic asthma mouse model. J Ethnopharmacol. 2018; 227:181-90.

https://doi.org/10.1016/j.jep.2018.08.028

PMID:30172058 